\title{
On Subordinations for Certain Multivalent Analytic Functions in the Right-Half Plane
}

\author{
Yi-Hui $X u^{1}$ and Jin-Lin Liu' \\ ${ }^{1}$ Department of Mathematics, Suqian College, Suqian 223800, China \\ ${ }^{2}$ Department of Mathematics, Yangzhou University, Yangzhou 225002, China
}

Correspondence should be addressed to Jin-Lin Liu; jlliu@yzu.edu.cn

Received 2 January 2016; Accepted 16 February 2016

Academic Editor: Gestur Ólafsson

Copyright (c) 2016 Y.-H. Xu and J.-L. Liu. This is an open access article distributed under the Creative Commons Attribution License, which permits unrestricted use, distribution, and reproduction in any medium, provided the original work is properly cited.

The object of the present paper is to investigate some properties of multivalent analytic functions associated with the lemniscate of Bernoulli.

\section{Introduction}

Let $A_{n}(p)$ denote the class of functions of the form

$$
f(z)=z^{p}+\sum_{k=n+p}^{\infty} a_{k} z^{k} \quad(p, n \in \mathbb{N}=\{1,2,3, \ldots\}),
$$

which are analytic in the open unit $\operatorname{disk} U=\{z:|z|<1\}$. A function $f \in A_{n}(p)$ is said to be starlike of order $\rho$ in $U$ if it satisfies

$$
\operatorname{Re}\left(\frac{z f^{\prime}(z)}{f(z)}\right)>\rho \quad(0 \leq \rho<p ; z \in U) .
$$

For functions $f$ and $g$ analytic in $U$, we say that $f$ is subordinate to $g$ and write $f(z) \prec g(z)(z \in U)$, if there exists an analytic function $w(z)$ in $U$ such that

$$
\begin{aligned}
|w(z)| & \leq|z|, \\
f(z) & =g(w(z)) \quad(z \in U) .
\end{aligned}
$$

Furthermore, if the function $g$ is univalent in $U$, then

$$
\begin{aligned}
f(z) & \prec g(z) \\
(z \in U) & \Longleftrightarrow f(0)=g(0), \\
f(U) & \subset g(U) .
\end{aligned}
$$

Let SL be the class of functions defined by

$$
\mathrm{SL}:=\left\{f \in A_{1}(1):\left|\left(\frac{z f^{\prime}(z)}{f(z)}\right)^{2}-1\right|<1\right\} .
$$

Thus a function $f \in$ SL if $z f^{\prime}(z) / f(z)$ lies in the region bounded by the right-half of the lemniscate of Bernoulli given by $\left|w^{2}-1\right|<1$. In terms of subordination, the class SL consists of normalized analytic functions $f$ satisfying $z f^{\prime}(z) / f(z) \prec \sqrt{1+z}$. This class SL was introduced by Sokól and Stankiewicz [1]. Several geometric properties had been investigated by Sokól and Stankiewicz [1]. They determined the radius of convexity for functions in the class SL. They also obtained structural formula, as well as growth and distortion theorems for these functions. Estimates for the first few coefficients of functions in SL were obtained in [2]. Recently, Sokól [3] determined various radii for functions belonging to the class SL; these include the radii of convexity, starlikeness, and strong starlikeness of order $\rho$. The SL-radii for certain well-known classes of functions including the Janowski starlike functions were obtained in [4]. General radii problems were also recently considered in $[5,6]$ wherein certain radii results for the class SL were obtained as special cases.

Motivated essentially by the above and some recent works [7], the main object of the present paper is to investigate some 
properties of multivalent analytic functions associated with the lemniscate of Bernoulli.

\section{Main Results}

The following lemma will be required.

Lemma 1 (see [8]). Let $q$ be univalent in the unit disk $U$ and let $\theta$ and $\varphi$ be analytic in a domain $D$ containing $q(U)$ with $\varphi(w) \neq 0$ when $w \in q(U)$. Set $Q(z)=z q^{\prime}(z) \varphi(q(z)), h(z)=$ $\theta(q(z))+Q(z)$. Suppose that

(1) either $h$ is convex or $Q$ is a starlike univalent function in $U$,

(2) $\operatorname{Re}\left(z h^{\prime}(z) / Q(z)\right)>0$ for $z \in U$.

If $p$ is analytic in $U, p(0)=q(0)$, and satisfies

$$
\begin{aligned}
& \theta(p(z))+z p^{\prime}(z) \varphi(p(z)) \\
& \quad \prec \theta(q(z))+z q^{\prime}(z) \varphi(q(z)),
\end{aligned}
$$

then $p(z) \prec q(z)$, and $q$ is the best dominant.

Theorem 2. Let $\alpha>0$. If $f \in A_{n}(p)$ satisfies

$$
\frac{f(z)}{z^{p}}\left(1+\alpha\left(\frac{z f^{\prime}(z)}{f(z)}-p\right)\right) \prec \sqrt{1+z} \quad(z \in U),
$$

then $f(z) / z^{p} \prec \sqrt{1+z}$.

Proof. Let $p(z)=f(z) / z^{p}$. Then $p(z)$ is analytic in $U$ with $p(0)=1$. Condition (7) becomes that

$$
\begin{aligned}
& \frac{f(z)}{z^{p}}\left(1+\alpha\left(\frac{z f^{\prime}(z)}{f(z)}-p\right)\right)=p(z)+\alpha z p^{\prime}(z) \\
& \quad \prec \sqrt{1+z} \quad(z \in U) .
\end{aligned}
$$

Define the function $q: U \rightarrow \mathbb{C}$ by $q(z)=\sqrt{1+z}$ with $q(0)=1$. Since $q(U)=\left\{w:\left|w^{2}-1\right|<1\right\}$ is the right-half of the lemniscate of Bernoulli, $q(U)$ is a convex set and hence $q$ is a convex function. This shows that the function $z q^{\prime}(z)$ is starlike with respect to the origin. We now show that $p(z) \prec$ $q(z)$ if the function $p$ satisfies the differential chain

$$
p(z)+\alpha z p^{\prime}(z) \prec q(z)+\alpha z q^{\prime}(z) .
$$

For this purpose, let the functions $\theta$ and $\varphi$ be defined by $\theta(w)=w$ and $\varphi(w)=\alpha$. Clearly the functions $\theta$ and $\varphi$ are analytic in $\mathbb{C}$ and $\varphi(w) \neq 0$. Also let $Q$ and $h$ be the functions defined by

$$
\begin{aligned}
Q(z) & =z q^{\prime}(z) \varphi(q(z))=\alpha z q^{\prime}(z), \\
h(z) & =\theta(q(z))+Q(z)=q(z)+\alpha z q^{\prime}(z) \\
& =\frac{2+(2+\alpha) z}{2 \sqrt{1+z}} .
\end{aligned}
$$

It is obvious that $Q$ is a starlike univalent function in $U$ and

$$
\operatorname{Re}\left\{\frac{z h^{\prime}(z)}{Q(z)}\right\}=\frac{1}{\alpha}+\operatorname{Re}\left\{1+\frac{z q^{\prime \prime}(z)}{q^{\prime}(z)}\right\}>0
$$

$(z \in U)$

for $\alpha>0$. By the lemma, it follows that $p \prec q=\sqrt{1+z}$. To complete the proof, it is left to show that

$$
q(z)=\sqrt{1+z} \prec \frac{2+(2+\alpha) z}{2 \sqrt{1+z}}=h(z) .
$$

Since $q^{-1}(w)=w^{2}-1$, it follows that

$$
q^{-1}(h(z))=z\left(1+\alpha+\frac{\alpha^{2}}{4(1+z)}\right) .
$$

For $z=e^{i t}, t \in[-\pi, \pi]$, clearly

$$
\left|q^{-1}\left(h\left(e^{i t}\right)\right)\right|=\left|1+\alpha+\frac{\alpha^{2}}{4\left(1+e^{i t}\right)}\right| .
$$

A simple calculation shows that the minimum of the above expression is attained at $t=0$. Hence

$$
\left|q^{-1}\left(h\left(e^{i t}\right)\right)\right| \geq 1+\alpha+\frac{\alpha^{2}}{8}>1 .
$$

Thus $q^{-1}(h(U)) \supset U$ or $h(U) \supset q(U)$. This shows that $q(z) \prec$ $h(z)$ and completes the proof.

Theorem 3. Let $f$ belong to the class $A_{n}(p)$ and satisfy

$$
\frac{f(z)}{z^{p}} \prec \sqrt{1+z} \quad(z \in U) .
$$

Then

$$
\operatorname{Re}\left((1-\delta)\left(\frac{f(z)}{z^{p}}\right)^{2}+\delta\left(\frac{z f^{\prime}(z)}{f(z)}\right)\right)>\rho
$$

$$
\left(|z|<r_{n}(\delta, \rho)\right),
$$

where $0 \leq \rho<p, \rho / p<\delta \leq 1$, and $r_{n}(\delta, \rho)$ is the smallest root in $(0,1)$ of the equation

$$
\begin{gathered}
2(1-\delta) r^{2 n}-[2(2-\rho)+\delta(n+2 p-4)] r^{n} \\
+2(1-\rho)+2 \delta(p-1)=0 .
\end{gathered}
$$

The result is sharp.

Proof. From (16) we can write

$$
\left(\frac{f(z)}{z^{p}}\right)^{2}=1+z^{n} \varphi(z)
$$

where $\varphi$ is analytic and $|\varphi(z)| \leq 1$ in $U$. Differentiating both sides of (19) logarithmically, we arrive at

$$
\frac{z f^{\prime}(z)}{f(z)}=p+\frac{n z^{n} \varphi(z)}{2\left(1+z^{n} \varphi(z)\right)}+\frac{z^{n+1} \varphi^{\prime}(z)}{2\left(1+z^{n} \varphi(z)\right)}
$$


Put $|z|=r<1$ and $\left(f(z) / z^{p}\right)^{2}=u+i v(u, v \in \mathbb{R})$. Then (19) implies that

$$
\begin{aligned}
z^{n} \varphi(z) & =u-1+i v, \\
1-r^{n} & \leq u \leq 1+r^{n} .
\end{aligned}
$$

With the help of the Carathéodory inequality

$$
\left|\varphi^{\prime}(z)\right| \leq \frac{1-|\varphi(z)|^{2}}{1-r^{2}}
$$

it follows from (21) that

$$
\begin{aligned}
& \operatorname{Re}\left((1-\delta)\left(\frac{f(z)}{z^{p}}\right)^{2}+\delta\left(\frac{z f^{\prime}(z)}{f(z)}\right)\right) \\
& \geq(1-\delta) u+p \delta+\frac{n \delta}{2} \operatorname{Re}\left(\frac{z^{n} \varphi(z)}{1+z^{n} \varphi(z)}\right) \\
& \quad-\frac{\delta}{2}\left|\frac{z^{n+1} \varphi^{\prime}(z)}{1+z^{n} \varphi(z)}\right| \\
& \geq(1-\delta) u+p \delta+\frac{n \delta}{2}\left(1-\frac{u}{u^{2}+v^{2}}\right) \\
& \quad+\frac{\delta}{2} \frac{(u-1)^{2}+v^{2}-r^{2 n}}{r^{n-1}\left(1-r^{2}\right)\left(u^{2}+v^{2}\right)^{1 / 2}}=F_{n}(u, v), \\
& \frac{\partial F_{n}(u, v)}{\partial v}=\delta v G_{n}(u, v),
\end{aligned}
$$

where $0<r<1,0<\delta \leq 1$, and

$$
\begin{aligned}
G_{n}(u, v)= & \frac{n u}{\left(u^{2}+v^{2}\right)^{2}}+\frac{1}{r^{n-1}\left(1-r^{2}\right)\left(u^{2}+v^{2}\right)^{1 / 2}} \\
& +\frac{r^{2 n}-\left((u-1)^{2}+v^{2}\right)}{2 r^{n-1}\left(1-r^{2}\right)\left(u^{2}+v^{2}\right)^{3 / 2}}>0
\end{aligned}
$$

because of (21). Since $F_{n}(u, v)$ is a dual function of $v$, from (23), (24), and (25), we see that

$$
\begin{aligned}
F_{n}(u, v) \geq & F_{n}(u, 0) \\
= & (1-\delta) u+p \delta+\frac{n \delta}{2}\left(1-\frac{1}{u}\right) \\
& +\frac{\delta\left((u-1)^{2}-r^{2 n}\right)}{2 r^{n-1}\left(1-r^{2}\right) u} .
\end{aligned}
$$

Now let us calculate the minimum value of $F_{n}(u, 0)$ on the closed interval $\left[1-r^{n}, 1+r^{n}\right]$. From (26) we deduce that

$$
\begin{aligned}
\frac{d}{d u} F(u, 0) & =1-\delta+\frac{\delta}{2 u^{2}}\left(n+\frac{r^{2 n}+u^{2}-1}{r^{n-1}\left(1-r^{2}\right)}\right) \\
& \geq 1-\delta+\frac{\delta}{2\left(1+r^{n}\right)^{2}}\left(n-\frac{2 r\left(1-r^{n}\right)}{1-r^{2}}\right) \\
& =1-\delta+\frac{\delta}{2\left(1+r^{n}\right)^{2}} I_{n}(r),
\end{aligned}
$$

where

$$
I_{n}(r)=n-\frac{2 r\left(1-r^{n}\right)}{1-r^{2}} .
$$

Note that $I_{1}(r)=(1-r) /(1+r)>0$. Suppose that $I_{n}(r)>0$. Then

$$
\begin{aligned}
I_{n+1}(r) & =n+1-\frac{2 r\left(1-r^{n+1}\right)}{1-r^{2}} \\
& =I_{n}(r)+\frac{\left(1-r^{n+1}\right)+r\left(1-r^{n}\right)}{1+r}>0 .
\end{aligned}
$$

Hence, by virtue of the mathematical induction, we have $I_{n}(r)>0$ for all $n \in \mathbb{N}$ and $0 \leq r<1$. This implies that

$$
\frac{d}{d u} F(u, 0)>0 \quad\left(1-r^{n} \leq u \leq 1+r^{n}\right) .
$$

Further it follows from (23), (26), and (30) that

$$
\begin{aligned}
\operatorname{Re}\left((1-\delta)\left(\frac{f(z)}{z^{p}}\right)^{2}+\delta\left(\frac{z f^{\prime}(z)}{f(z)}\right)\right)-\rho & \geq F_{n}\left(1-r^{n}, 0\right)-\rho=(1-\delta)\left(1-r^{n}\right)+p \delta-\frac{n \delta r^{n}}{2\left(1-r^{n}\right)}-\rho \\
& =\frac{2(1-\delta) r^{2 n}-[2(2-\rho)+\delta(n+2 p-4)] r^{n}+2(1-\rho)+2 \delta(p-1)}{2\left(1-r^{n}\right)} \\
& =\frac{J_{n}(r)}{2\left(1-r^{n}\right)},
\end{aligned}
$$

where

$$
\begin{aligned}
J_{n}(r)= & 2(1-\delta) r^{2 n}-[2(2-\rho)+\delta(n+2 p-4)] r^{n} \\
& +2(1-\rho)+2 \delta(p-1) .
\end{aligned}
$$

Note that $J_{n}(0)=2(1-\rho)+2 \delta(p-1)>0$ and $J_{n}(1)=$ $-n \delta<0$. If we let $r_{n}(\delta, \rho)$ denote the root in $(0,1)$ of the equation $J_{n}(r)=0$, then (31) yields the desired result (17). 
To see that the bound $r_{n}(\delta, \rho)$ is the best possible, we consider the function

$$
f(z)=z^{p}\left(1-z^{n}\right)^{1 / 2}
$$

Clearly the function $f$ defined by (33) satisfies condition (16). Also it is obvious that, for $z=r \in\left(r_{n}(\delta, \rho), 1\right)$,

$$
\begin{aligned}
& (1-\delta)\left(\frac{f(r)}{r^{p}}\right)^{2}+\delta\left(\frac{r f^{\prime}(r)}{f(r)}\right)-\rho=\frac{J_{n}(r)}{2\left(1-r^{n}\right)} \\
& \quad<0
\end{aligned}
$$

which shows that the bound $r_{n}(\delta, \rho)$ can not be increased. The proof of the theorem is completed.

Setting $\delta=1$, Theorem 3 reduces to the following.

Corollary 4. Let $f$ belong to the class $A_{n}(p)$ and satisfy condition (16). Then $f$ is starlike of order $\rho(0 \leq \rho<p)$ in

$$
|z|<\left(\frac{2(p-\rho)}{2(p-\rho)+n}\right)^{1 / n} .
$$

The result is sharp.

\section{Competing Interests}

The authors declare that there are no competing interests regarding the publication of this paper.

\section{Acknowledgments}

This research is supported by the National Natural Science Foundation of China (no. 11571299) and the Natural Science Foundation of Jiangsu Province (no. BK20151304). The work is also supported by the Natural Science Foundation of Suqian College (no. 2015KY25).

\section{References}

[1] J. Sokól and J. Stankiewicz, "Radius of convexity of some subclasses of strongly starlike functions," Zeszyty Naukowe Politechniki Rzeszowskiej. Matematyka, vol. 19, pp. 101-105, 1996.

[2] J. Sokól, "Coefficient estimates in a class of strongly starlike functions," Kyungpook Mathematical Journal, vol. 49, no. 2, pp. 349-353, 2009.

[3] J. Sokól, "Radius problems in the class SL", Applied Mathematics and Computation, vol. 214, no. 2, pp. 569-573, 2009.

[4] R. M. Ali, N. K. Jain, and V. Ravichandran, "Radii of starlikeness associated with the lemniscate of Bernoulli and the left-half plane," Applied Mathematics and Computation, vol. 218, no. 11, pp. 6557-6565, 2012.

[5] R. M. Ali, N. E. Cho, V. Ravichandran, and S. S. Kumar, "Differential subordination for functions associated with the lemniscate of Bernoulli," Taiwanese Journal of Mathematics, vol. 16, no. 3, pp. 1017-1026, 2012.

[6] R. M. Ali, N. E. Cho, N. K. Jain, and V. Ravichandran, "Radii of starlikeness and convexity for functions with fixed second coefficient defined by subordination," Filomat, vol. 26, no. 3, pp. 553-561, 2012.
[7] D.-G. Yang and J.-L. Liu, "On a class of analytic functions with missing coefficients," Applied Mathematics and Computation, vol. 215, no. 9, pp. 3473-3481, 2010.

[8] S. S. Miller and P. T. Mocanu, Differential Subordination, Theory and Application, Marcel Dekker, New York, NY, USA, 2000. 


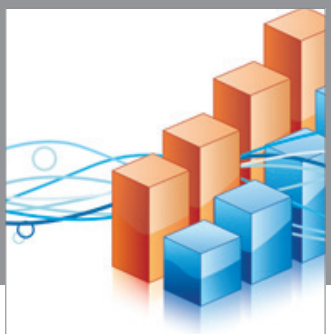

Advances in

Operations Research

vatem alat4

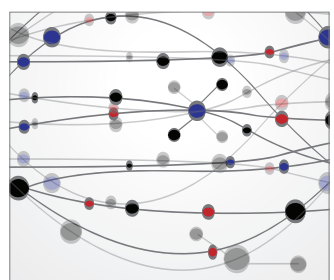

\section{The Scientific} World Journal
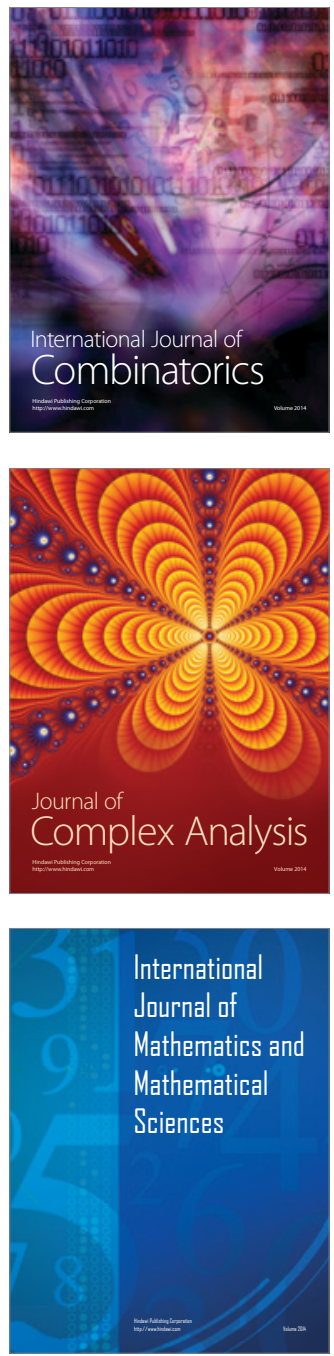
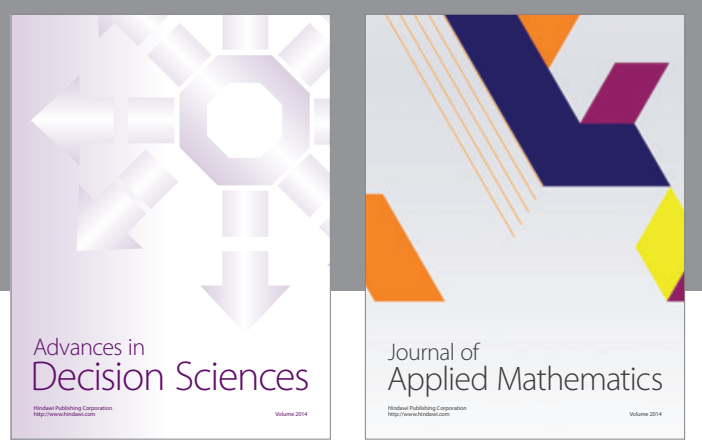

Algebra

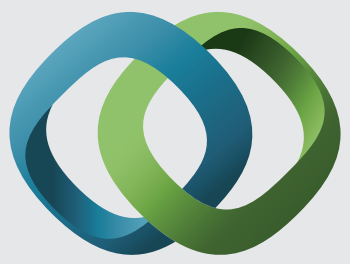

\section{Hindawi}

Submit your manuscripts at

http://www.hindawi.com
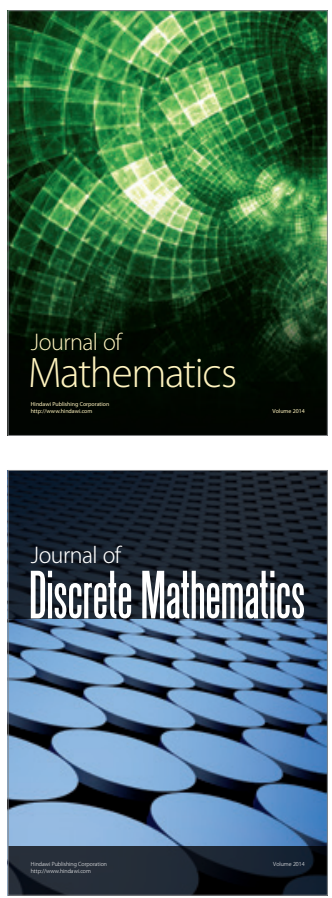

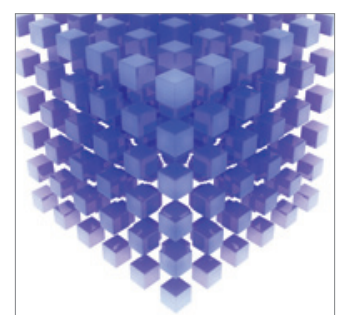

Mathematical Problems in Engineering
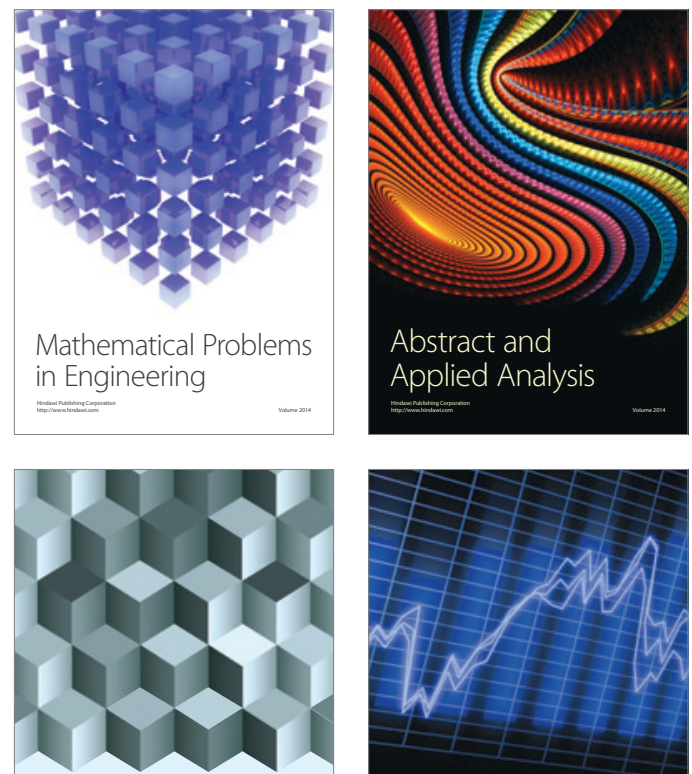

Journal of

Function Spaces

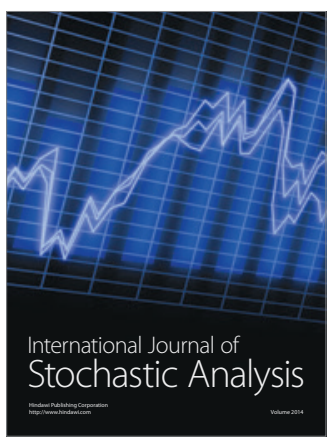

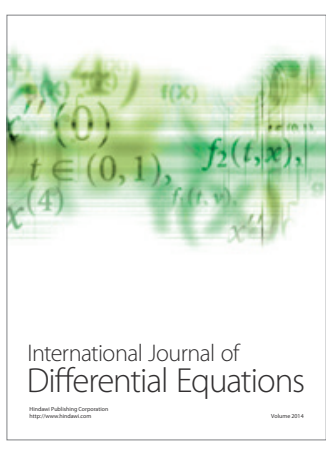
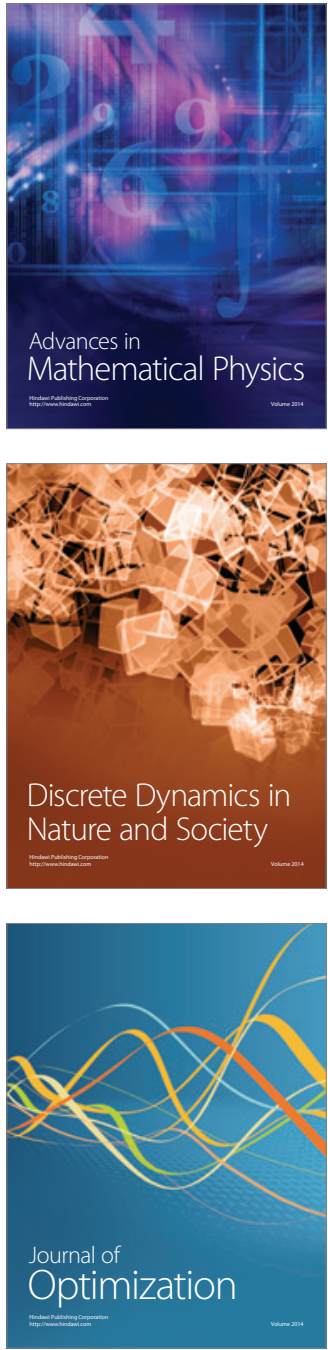